\title{
Electron diffraction studies of hot molecules. III. Stretching and bending anharmonicity in $\mathrm{CF}_{3} \mathrm{Cl}$
}

\author{
Lawrence S. Bartell, William N. Vance, and Steven R. Goates ${ }^{a)}$ \\ Department of Chemistry, University of Michigan, Ann Arbor, Michigan 48109
}

(Received 14 December 1983; accepted 20 January 1984)

\begin{abstract}
Bond lengths, amplitudes of vibration, and asymmetries in distribution of nonbonded atom pairs were determined for $\mathrm{CF}_{3} \mathrm{Cl}$ at temperatures ranging from 300 to $1220 \mathrm{~K}$. The $\mathrm{CF}$ but not the $\mathrm{CCl}$ bond length followed the commonly invoked approximation $\Delta r=(3 a / 2) \Delta l^{2}+\Delta K+\Delta \delta r$. The coefficients of skewness of nonbonded radial distribution peaks revealed substantial bending anharmonicity comparable to that detected in a previous study of binary fluorides and accounted for by the valence-shell-electron-pair repulsion force field in its points-on-a-sphere representation.
\end{abstract}

\section{INTRODUCTION}

In the course of an electron diffraction investigation of hot polyatomic molecules,' useful new perspectives about stretching and bending anharmonicity were gained. Of particular significance was the discovery, ${ }^{2,3}$ subsequently corroborated by molecular orbital calculations, ${ }^{4}$ that a simple mechanical model affords a rather good representation of the potential surface for bending deformations. Because this model, based on the valence-shell-electron-pair repulsion theory, ${ }^{5}$ is easy to apply and appears to offer useful predictive powers, further experimental investigation seemed appropriate.

A major incentive for initiating the study of hot molecules, in the first place, had been the serendipitous invention ${ }^{1}$ of a laser-heated micronozzle providing several advantages over conventional oven nozzles. Because all of the molecules so far studied by this technique have been binary compounds $\left(\mathrm{AX}_{n}\right)$, each with a single type of bond, it appeared worthwhile to analyze an example possessing more than one kind of bond. An obvious selection was $\mathrm{CF}_{3} \mathrm{Cl}$, a molecule distinguished by displaying as well resolved a spectrum of internuclear distances as ever seen in a molecule with more than three kinds of distances. ${ }^{6}$ This molecule has been the subject of three prior sectored electron diffraction studies. ${ }^{6-8}$ Since all of them were carried out at room temperature, amplitudes of vibrations had been too small to sample the highly anharmonic region of the potential energy surface and little had been learned about anharmonicity. The present investigation undertaken as an undergraduate project, while not achieving thermal excitations comparable to the highest previously reported, ${ }^{1}$ nevertheless attained temperatures exceeding $1200 \mathrm{~K}$ and revealed clear manifestations of anharmonicity. Analyses are reported in the following.

\section{EXPERIMENTAL}

A sample of $\mathrm{CF}_{3} \mathrm{Cl}$ with a stated purity in excess of $99 \%$ purchased from Matheson Gas Products was used as obtained. Gas at 200 Torr was passed through a stainless steel

\footnotetext{
^) Present address: Department of Chemistry, Brigham Young University,
} Provo, Utah 84602. nozzle $6 \mathrm{~mm}$ long and $0.1 \mathrm{~mm}$ in i.d., constructed from a hypodermic needle, into the electron diffraction apparatus. Heating was accomplished by focusing the output of a $40 \mathrm{~W}$ $\mathrm{cw} \mathrm{CO}_{2}$ laser onto the micronozzle close to the tip. Powers were adjusted from 0 to $37 \mathrm{~W}$ to achieve various visibly discernible levels of incandescence ranging from red through brilliant yellow. Procedures closely followed those of Ref. 1 . Gas transmitted through the $40 \mathrm{kV}$ electron beam was condensed on a large surface cooled by liquid nitrogen. Diffraction exposures ranged from $3.4 \mathrm{~s}$ for $300 \mathrm{~K}$ plates to $10 \mathrm{~s}$ for the hottest. Patterns were recorded through an $r^{3}$ sector on $4 \times 5$ in. Kodak electron image plates $21 \mathrm{~cm}$ from the nozzle.

\section{ANALYSIS OF DATA}

Procedures followed those outlined in Refs. 1 and 9 both in the processing of diffraction plates and refinement of diffraction intensities. Among other things, experimental intensities from the hot plates were corrected for intramolecular multiple scattering ${ }^{10}$ (computed to correspond to ambient temperature) and for the systematic residuals $\overline{\Delta M}_{r}(s)$ of the control plates as justified elsewhere. ${ }^{1,9}$ Residuals for the three control plates adopted as representative are plotted in Fig. 1. Their practical significance is that, when they are employed as corrections, intensity residuals of the hot plates are considerably reduced and become more or less random. While this does not increase the absolute accuracy of the least squares refinements, it does enable the precision of the thermal effects sought to be inferred easily. A potential theoretical significance of the residuals is that they contain information about the redistribution of electronic charge in the molecule away from that implied by the independent atom model used in refinements. Such an effect of bond formation was proposed ${ }^{9}$ to account for analogous residuals observed in the case of $\mathrm{SF}_{6}$. This interpretation was subsequently confirmed by Pulay et al. ${ }^{11}$ who calculated the electron density distribution in $\mathrm{SF}_{6}$ by ab initio molecular orbital theory.

Temperatures were deduced by comparing observed nonbonded amplitudes of vibration with those calculated as a function of temperature with the Hilderbrandt routine MSAV ${ }^{12}$ after the systematic "offsets" of mean-square amplitudes at $300 \mathrm{~K}$ had been established. ${ }^{1,9,13}$ Computations 


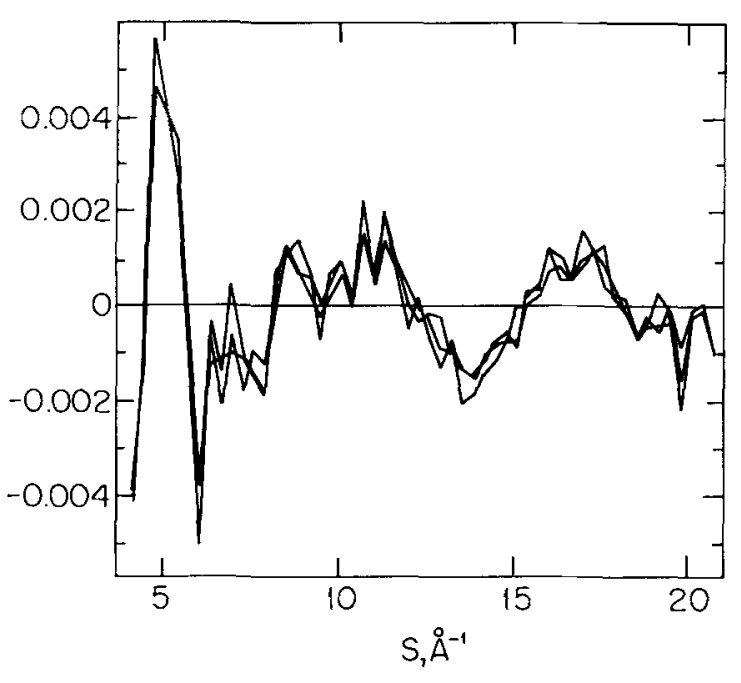

FIG. 1. Superposition of the residuals $\Delta M_{r}(s)$ of the three control plates of $\mathrm{CF}_{3} \mathrm{Cl}$ after correcting for intramolecular multiple scattering. Ordinate $\Delta M$, represents the ratio $\left(I_{\text {expt }}-I_{\text {calc }}\right) / I_{\text {expt }}$.

were based on the force field of Taylor. ${ }^{14}$ Nonbonded amplitudes were chosen for comparison because they are more sensitive to temperature changes than are bonded amplitudes. The average difference between temperatures implied by the $\mathrm{FF}$ and $\mathrm{FCl}$ amplitudes of individual plates was about $40^{\circ}$ with the FF amplitude systematically hotter by this amount (through some artifact). A geometric average temperature was adopted for each plate.

Some refinements were carried out fitting the four separate internuclear distances independently. The most definitive analyses, however, incorporated temperature-dependent theoretical shrinkages while varying $r_{\mathrm{CF}}, r_{\mathrm{CCl}}$, and $\angle \mathrm{FCF}$, maintaining $C_{3 v}$ symmetry. Bond asymmetry constants $\hat{a}(T),{ }^{3}$ listed in part in Table $\mathrm{I}$, were calculated according to Ref. 15, using the Morse-like constants of Herschbach and Laurie, ${ }^{16}$ and nonbonded $\hat{a}$ values ${ }^{3}$ were preset to various values. Preferred values were those giving the smallest standard deviations in least squares refinements.
TABLE I. Bond asymmetry parameters ${ }^{a}$ according to method of Ref. 15.

\begin{tabular}{llllll}
\hline \hline$T$ & 0 & 300 & 600 & 900 & 1200 \\
\hline${ }^{a} \mathrm{CF}$ & 2.01 & 2.07 & 2.86 & 3.84 & 4.53 \\
${ }^{a} \mathrm{CCl}$ & 1.82 & 2.14 & 3.44 & 4.32 & 4.78 \\
\hline \hline
\end{tabular}

an $\AA^{-1}$, temperature in $\mathrm{K}$.

\section{RESULTS}

The aim of this research is to study the effect of thermal excitation on molecules and to extract some information about anharmonicity. To do this, reference molecular lengths and amplitudes at room temperature must first be established. Here it should be noted that experimental constraints dictated by the unconventional requirements of the measurements made it unfeasible to record intensities out to high scattering angles. This limitation reduced, somewhat, the absolute accuracy in derived parameters owing to the systematic errors known to be disproportionately associated with small angle data. As explained elsewhere, ${ }^{1,9}$ however, this source of trouble has little effect on the precision of the derived parameters and, hence, interferes little with the measurements of changes in molecular parameters with heating. For sake of illustration, however, we compare in Table II our $300 \mathrm{~K}$ internuclear distances and amplitudes with those derived in three other electron diffraction analyses of $\mathrm{CF}_{3} \mathrm{Cl}$, all of which included higher angle data. ${ }^{68}$ It is seen that, despite our limitation, our internuclear distances and amplitudes agree well with those of two of the three other studies. Results of Ref. 7 which, according to listed standard deviations are the most accurate, are somewhat out of line with the others and with spectroscopic amplitudes.

Experimental leveled intensities are plotted in Fig. 2 for $\mathrm{CF}_{3} \mathrm{Cl}$ at various temperatures. Effects of thermal excitation are evident in the damping of molecular interference features. Results can most easily be understood by referring to the four internuclear peaks $\mathrm{C}-\mathrm{F}, \mathrm{C}-\mathrm{Cl}, \mathrm{F} \ldots \mathrm{F}$, and $\mathrm{F} \ldots \mathrm{Cl}$, displayed in the radial distribution function of Fig. 3 calculated from the $300 \mathrm{~K}$ electron diffraction intensities of Ref. 6. (These data were selected for illustrative purposes rather than the present data because of the aforementioned limitation imposed by the present experimental constraints.) Mea-

TABLE II. Electron diffraction analyses ${ }^{\mathrm{a}}$ of $\mathrm{CF}_{3} \mathrm{Cl}$ at $300 \mathrm{~K}$

\begin{tabular}{|c|c|c|c|c|c|c|c|}
\hline & $\mathbf{T D O}+\mathbf{M W}^{\mathbf{b}}$ & $\mathrm{TDO}^{\mathrm{b}}$ & $\mathbf{B B}^{\mathbf{c}}$ & $\mathbf{J H}^{\mathrm{d}}$ & $\mathrm{BVG}^{\mathrm{e}}$ & $\operatorname{Spec}^{f}$ & Spec ${ }^{\mathrm{g}}$ \\
\hline$r_{\mathrm{CF}}$ & $1.327(0.6)$ & $1.328(0.5)$ & $1.330(2)$ & $1.329(1)$ & $1.327(1)\left[0.1_{5}\right]$ & & \\
\hline$r_{\mathrm{CCl}}$ & $1.754(1.5)$ & $1.751(1)$ & $1.754(4)$ & $1.757(2)$ & $1.760(3)[0.4]$ & & \\
\hline$r_{F F}$ & $\cdots$ & $2.152(0.6)$ & $2.162(3)$ & $2.157(1.5)$ & $2.157(1)[0.2]$ & & \\
\hline$r_{\mathrm{CIF}}$ & --- & $2.531(0.5)$ & $2.545(3)$ & $2.542(1)$ & $2.540(1)[0.2]$ & & \\
\hline$\angle \mathrm{FCF}$ & $108.6(1)$ & --- & $108.6(3)$ & $108.7(1)$ & $108.8(1)[0.3]$ & & \\
\hline$l_{\mathrm{CF}}$ & $0.056(1)$ & $0.055(1)$ & $0.049(2)$ & $0.049(2)$ & $0.046(4)[0.5]$ & 0.045 & 0.045 \\
\hline$l_{\mathrm{CCl}}$ & $0.063(2)$ & $0.062(1)$ & $0.054(5)$ & $0.053(2)$ & $0.053(6)[0.8]$ & 0.046 & 0.050 \\
\hline$l_{\mathrm{FF}}$ & $0.066(1)$ & $0.065(1)$ & $0.058(3)$ & $0.060(2)$ & $0.053(3)[0.5]$ & 0.054 & 0.054 \\
\hline$l_{\mathrm{CIF}}$ & $0.076(1)$ & $0.075(1)$ & $0.066(3)$ & $0.067(1.5)$ & $0.065(2)[0.3]$ & 0.060 & 0.064 \\
\hline
\end{tabular}

${ }^{\text {a Distance, } r} r_{\mathrm{g}}$ in $\AA$; uncertainties in parentheses, standard deviations. ${ }^{\mathrm{b}}$ Reference 7 results transformed to present basis. ${ }^{\mathrm{c}}$ Reference 6 , converted to modern parameters. ${ }^{d}$ Reference $8, \hat{a}=2$ applied to nonbonded distance. ${ }^{\circ}$ This work. Parentheses indicate accuracies; square brackets, precisions, three- to eight fold better than accuracies. The difference arises from the systematic residuals $\Delta M_{r}$ (see the text) that distort refinements and that are largely attributable to inaccuracies in electron densities of the independent atom model used. ${ }^{f}$ This work applying force field in Ref. $14 .{ }^{8}$ Computed from force field of $A$. Ruoff by H. Oberhammer (private communication, 1984). 


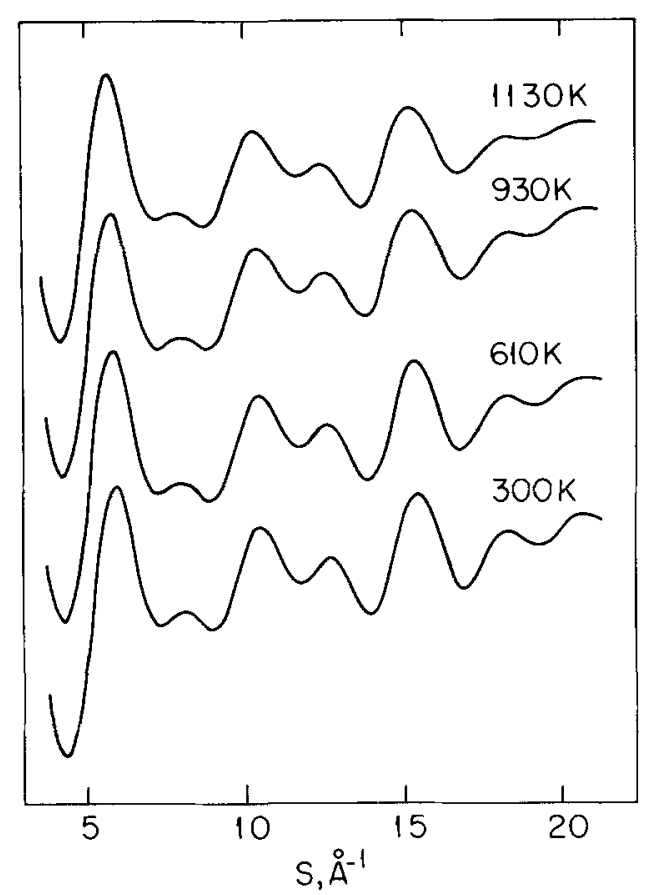

FIG. 2. Experimental leveled intensities of $\mathrm{CF}_{3} \mathrm{Cl}$ at various temperatures.

sured increases in amplitudes of vibration $\Delta l_{i j}$ as the sample is heated beyond $300 \mathrm{~K}$ are displayed in Fig. 4 . Here the increases in nonbonded amplitudes are compared with the increases in corresponding bond amplitudes. Among other things, Fig. 4 demonstrates that bending amplitudes of $\mathrm{CF}_{3} \mathrm{Cl}$ are a more sensitive measure of temperature than are stretching amplitudes.

Thermal excitation increases bond lengths, as well as amplitudes of vibration, as shown in Fig. 5. How well the increases agree with a commonly invoked approximation will be discussed in the next section.

Finally, an examination of least squares refinements with various nonbonded asymmetry constants ${ }^{3}$ indicated that in $\AA^{-1}$,

$$
\hat{a}_{\mathrm{FCl}} \approx 2.7+0.2 \Delta
$$

and

$$
\hat{a}_{\mathrm{FF}} \approx 2.7-0.8 \Delta,
$$

with standard deviations perhaps $0.3 \AA^{-1}$. The two asym-

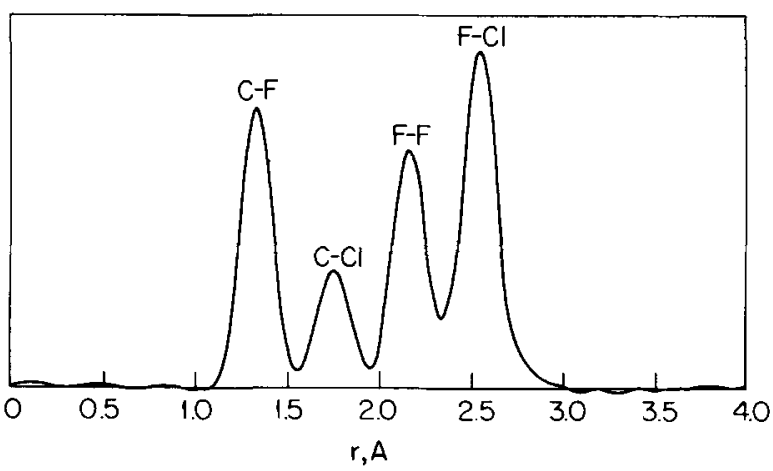

FIG. 3. Experimental radial distribution for $\mathrm{CF}_{3} \mathrm{Cl}$, Degard constant $0.0023 \AA$, data of Ref. 6 .

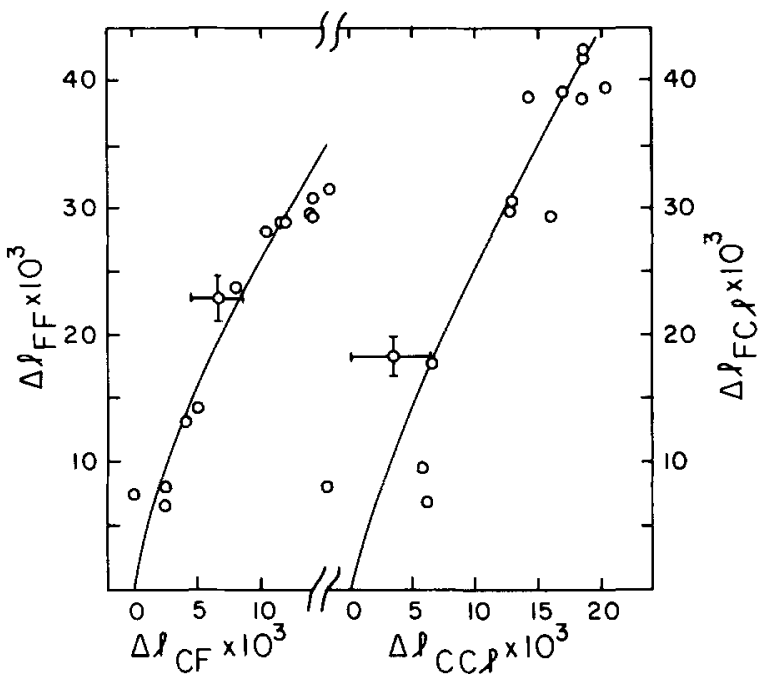

FIG. 4. Thermally induced increase in rms nonbonded amplitudes of vibration of $\mathrm{CF}_{3} \mathrm{Cl}$ vs corresponding increase in bond amplitudes. Error bars, representative precision $( \pm 3 \sigma)$ of refinements corrected for residuals of Fig. 1. Solid lines, normal coordinate theory.

metry constants are correlated by the diffraction data as expressed by the parameter $\Delta$, whose value is very poorly established by the experiment. Our subjective guess is that

$$
\Delta \approx 1 \pm 1
$$

In the hotter runs there is no discernible variation of $\hat{a}$ with temperature. At lower temperatures intensities are insensitive to $\hat{a}$.

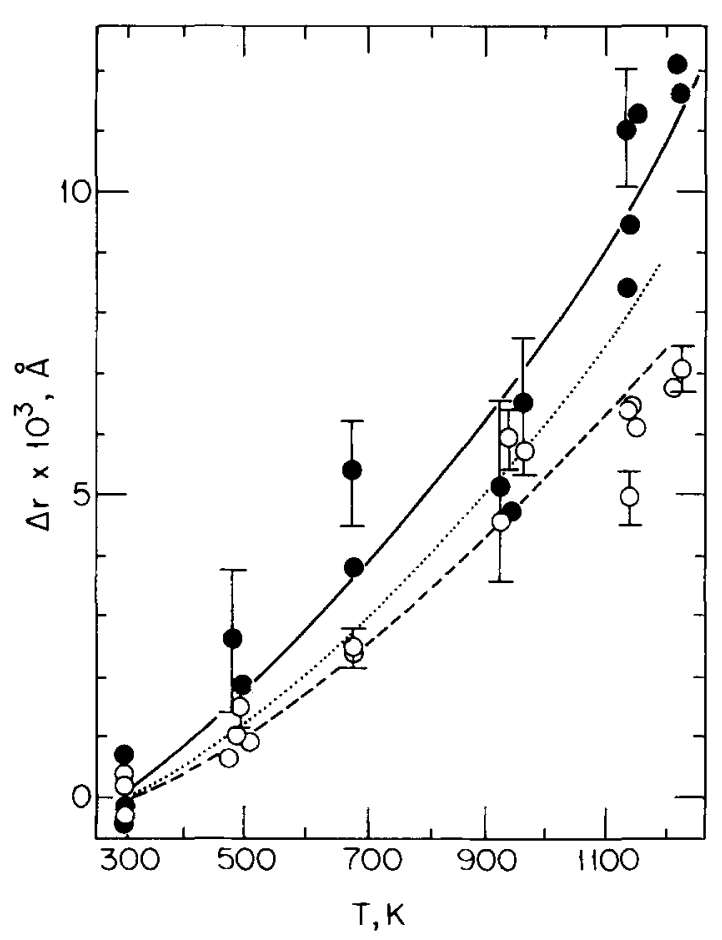

FIG. 5. Thermal expansion of bond lengths of $\mathrm{CF}_{3} \mathrm{Cl}$. Open points, C-F; dashed line, Eq. (4) for C-F. Solid points, $\mathrm{C}-\mathrm{Cl}$; solid line, smooth curve through solid points; dotted line, Eq. (4) for C-Cl. Error bars for selected points represent precision $( \pm 3 \sigma)$ of refinements corrected for residuals of Fig. 1. 


\section{DISCUSSION}

A puzzling aspect of the first diffraction study of the response of molecules to extreme changes in temperature ${ }^{1}$ was observed in plots of nonbonded vs bond amplitudes (cf. Fig. 4). It was found that, as the temperature was raised, experimental points fell increasingly to the right of the curve calculated by normal coordinate theory. That is, temperatures of stretching modes seemed to become hotter than those of bending modes. Subsequently, ${ }^{17}$ it was found that when $\hat{a}$ values were allowed to refine for nonbonded peaks, the vibrational amplitudes of the bonds shifted in such a way as to bring stretches into harmony with bends. This unexpected correlation between bonded and nonbonded parameters illustrates how errors can be introduced into structure refinements if nonbonded asymmetry is neglected. In the present work, points in Fig. 4 were determined after finding the $\hat{a}$ values and, within the appreciable experimental uncertainties, no incompatibility between stretching and bending temperatures is discernible. A small systematic discrepancy between the two types of nonbonded amplitudes was, however, seen as mentioned previously.

A direct measure of stretching anharmonicity is provided by the thermal expansion of the covalent bonds conspicuously apparent in Fig. 5 . Several procedures ${ }^{18,19}$ have been developed to relate bond lengths to vibrational energy if the anharmonic force field is known. Moreover, a simple treatment $^{20}$ has been successful for binary compounds $\left(\mathrm{AX}_{n}\right)$ where little more than the quadratic field and the (Herschbach-Laurie ${ }^{16}$ ) Morse parameter are known. This treatment is based on an extrapolation of the Urey-Bradley (UB) field to cubic terms by a simple recipe. Unfortunately, neither the full anharmonic field nor the equations for the anharmonic $\mathrm{UB}$ treatment are available for $\mathrm{CF}_{3} \mathrm{Cl}$. A yet simpler relation between bond length and amplitudes of vibration is often invoked, ${ }^{21}$ namely (neglecting centrifugal distortion),

$$
r_{T}-r_{r}=(3 / 2) a\left(l_{T}^{2}-l_{r}^{2}\right)+\left(K_{T}-K_{r}\right),
$$

where subscripts $T$ and $r$ indicate quantities at temperature $T$ and at some reference temperature, $a$ is the Morse diatomic parameter, ${ }^{22}$ and $K$ is given by

$$
\left.K=\left(\left\langle(\Delta x)^{2}\right\rangle+\langle\Delta y)^{2}\right\rangle\right) / 2 r
$$

expressing the effect of perpendicular amplitudes of vibration. ${ }^{23}$

The origin of Eq. (4) and its deficiencies are discussed in some detail in Ref. 1. In the absence of more definitive information the equation is a rational first approximation. When it gives a good representation of experimental data, as it often does, it is due in some measure to an accidental cancellation of errors. In the particular case of $\mathrm{CF}_{3} \mathrm{Cl}$, the experimental points for $r_{\mathrm{CF}}$ do follow $\mathrm{Eq}$ (4) reasonably well (as they also did for $r_{C F}$ in $\mathrm{CF}_{4}$ in Ref. 1). On the other hand, experimental $\mathrm{C}-\mathrm{Cl}$ bond lengths, while less well determined in $\mathrm{CF}_{3} \mathrm{Cl}$ than their fluorine analogs, appear to rise markedly faster with temperature than the dotted curve of Eq. (4). Deviations in the opposite direction from Eq. (4) had been ob- served for $\mathrm{SiF}_{4}{ }^{1}$ a molecule conforming well to the model UB extrapolation. It would be of some interest to find whether the $\mathrm{C}-\mathrm{Cl}$ result can be accounted for by the UB model.

Separate determinations of the $\mathrm{F} . . \mathrm{F}$ and $\mathrm{F}$... Cl skew parameters $\hat{a}$ were, regretably, not possible from the present experiment. If temperatures $500^{\circ}$ hotter had been attained as in the previous research, ${ }^{1}$ the parameter correlation frustrating this resolution might have been broken. As it is, the values implied by Eqs. (1) and (2) are consistent with previous results. For $\mathrm{CF}$, theoretical calculations of $\hat{a}_{\mathrm{FF}}$ in the present temperature range yielded the value of $2.1 \AA^{-1}$. Such a result inserted into Eqs. (1) and (2) would imply $\hat{a}_{\mathrm{FCl}} \approx 2.9 \AA^{-1}$, a value slightly higher than that of the cis $\mathrm{F}$...F pair in $\mathrm{SF}_{6} .{ }^{17}$ In any event, the $\hat{a}$ values show that bending modes in $\mathrm{CF}_{3} \mathrm{Cl}$ exhibit a decided anharmonicity in the direction seen in binary fluorides. ${ }^{1,3}$ As described elsewhere, ${ }^{2-4}$ the simple mechanical model alluded to in the Introduction has been effective in accounting for harmonic and anharmonic force constants for bending, as well as for structures of binary compounds. Whether the model can be successfully extended to examples of lower symmetry such as $\mathrm{CF}_{3} \mathrm{Cl}$ is a subject for future investigation.

\section{ACKNOWLEDGMENTS}

This research was supported by a grant from the National Science Foundation. We thank the Michigan Computing Center for a generous allocation of computing time.

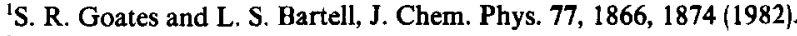

${ }^{2}$ L. S. Bartell, Croat. Chem. Acta (in press).

${ }^{3}$ L. S. Bartell, J. Mol. Struct. 84, 117 (1982). Skewness parameter $\hat{a}$ for an internuclear distribution is the ratio of moments $\left((x-\langle x\rangle)^{3}\right\rangle /$ $\left\langle\left(x-\left.\langle x\rangle\right|^{2}\right\rangle^{2}\right.$

${ }^{4}$ L. S. Bartell and Y. Barshad (umpublished research).

${ }^{5}$ R. J. Gillespie, Molecular Geometry (Van Nostrand, London, 1972).

${ }^{6}$ L. S. Bartell and L. O. Brockway, J. Chem. Phys. 23, 1860 (1955).

${ }^{7}$ V. Typke, M. Dakkouri, and H. Oberhammer, J. Mol. Struct. 44, 85 (1978).

${ }^{8}$ E. J. Jacob and K. Hedberg (private communication, 1978).

${ }^{9}$ L. S. Bartell, M. A. Kacner, and S. R. Goates, J. Chem. Phys. 75, 2730, 2736 (1981).

${ }^{10}$ B. R. Miller and L. S. Bartell, J. Chem. Phys. 72, 800 (1980).

"P. Pulay, R. Mawhorter, D. A. Koh1, and M. Fink, J. Chem. Phys. 79, 185 (1983)

${ }^{12}$ R. L. Hilderbrandt and J. D. Weiser, J. Chem. Phys. 55, 4648 (1971)

${ }^{13}$ M. H. Kelley and M. Fink, J. Chem. Phys. 77, 1813 (1982).

${ }^{14}$ R. C. Taylor, J. Chem. Phys. 22, 714 (1954).

${ }^{15}$ K. Kuchitsu, Bull. Chem. Soc. Jpn. 40, 498 (1967),

${ }^{16}$ D. R. Herschbach and V. W. Laurie, J. Chem. Phys. 35, 458 (1961).

${ }^{17}$ J. F. Stanton and L. S. Bartell (unpublished research).

${ }^{18}$ R. L. Hilderbrandt and D. A. Kohl, Theochemistry 2, 25 (1981); D. A Kohl and R. L. Hilderbrandt, ibid. 2, 325 (1981).

${ }^{19}$ K. Kuchitsu and L. S. Bartell, J. Chem. Phys. 36, 2460, 2470 (1962); I. M. Mills, J. Phys. Chem. 80, 1187 (1976).

${ }^{20}$ L. S. Bartell, J. Chem. Phys. 70, 4581 (1979).

${ }^{2}$ 'See, for example, K. Kuchitsu, J. Chem. Phys. 44, 906 (1966); J. Mol. Struct. 4, 41 (1969).

${ }^{22}$ L. S. Bartell, J. Chem. Phys. 23, 1219 (1955).

${ }^{23}$ See S. J. Cyvin, Molecular Vibrations and Mean Square Amplitudes (Elsevier, Amsterdam, 1968) 